\title{
REVIEW
}

\section{Left atrial appendage: structure, function, and role in thromboembolism}

\author{
N M Al-Saady, O A Obel, A J Camm
}

\begin{abstract}
The left atrial appendage (LAA) is derived from the left wall of the primary atrium, which forms during the fourth week of embryonic development. It has developmental, ultrastructural, and physiological characteristics distinct from the left atrium proper. The LAA lies within the confines of the pericardium in close relation to the free wall of the left ventricle and thus its emptying and filling may be significantly affected by left ventricular function. The physiological properties and anatomical relations of the LAA render it ideally suited to function as a decompression chamber during left ventricular systole and during other periods when left atrial pressure is high. These properties include the position of the LAA high in the body of the left atrium; the increased distensibility of the LAA compared with the left atrium proper; the high concentration of atrial natriuretic factor (ANF) granules contained within the LAA; and the neuronal configuration of the LAA. Thrombus has a predilection to form in the LAA in patients with atrial fibrillation, mitral valve disease, and other conditions. The pathogenesis has not been fully elucidated; however, relative stasis which occurs in the appendage owing to its shape and the trabeculations within it is thought to play a major role. Obliteration or amputation of the LAA may help to reduce the risk of thromboembolism, but this may result in undesirable physiological sequelae such as reduced atrial compliance and a reduced capacity for ANF secretion in response to pressure and volume overload.
\end{abstract}

(Heart 1999;82:547-555)Keywords: left atrial appendage; atrial fibrillation; thromboembolism

In the past, the left atrial appendage (LAA) has been considered to be a relatively insignificant portion of cardiac anatomy. It is now recognised that it is a structure with important pathological associations. ${ }^{1}$ First, thrombus has a predilection to form within the LAA in patients with non-valvar atrial fibrillation and to a lesser extent in those with mitral valve dis- ease (both in atrial fibrillation and in sinus rhythm). Second, the use of transoesophageal echocardiography has made clear imaging of the LAA possible, so that its size, shape, flow patterns, and content can be assessed in health and disease.

The risk of stroke is increased approximately fivefold in non-rheumatic atrial fibrillation ${ }^{2}$ and 17 -fold in patients with mitral stenosis and atrial fibrillation. ${ }^{3}$ About $15 \%$ of ischaemic strokes arise as a result of atrial fibrillation. ${ }^{4}$ Approximately $90 \%$ of atrial thrombi in non-rheumatic atrial fibrillation and $60 \%$ of such thrombi in patients with rheumatic mitral valve disease (predominantly stenosis) are seen within the LAA. ${ }^{5}$

Although the incidence of thromboembolism in atrial fibrillation and mitral valve disease can be dramatically reduced with the use of anticoagulants, particularly warfarin, the use of such treatment can be complicated, and is contraindicated in many patients. ${ }^{6}$ Alternative forms of treatment are needed for the prophylaxis of thromboembolism in these patients.

In this paper, we will discuss the ultrastructure and physiological function of the LAA and describe the local anatomical and physiological correlates associated with LAA thrombus. Finally, prophylaxis against thromboembolism by obliterating or amputating the LAA will be discussed.

\section{Anatomy}

The trabecular LAA is the remnant of the original embryonic left atrium that develops during the third week of gestation. ${ }^{7}$ The main smooth walled left atrial cavity develops later and is formed from the outgrowth of the pulmonary veins.

The LAA is a long, tubular, hooked structure which is usually crenellated and has a narrow junction with the venous component of the atrium. In contrast, the right appendage is broad and triangular with a wide junction. Unlike on the right, the junction of the LAA with the atrium proper is not marked either externally or internally by a crest or groove. Both the right and left appendages are trabeculated, with muscle bars largely running parallel to each other giving a comb-like appearance (hence termed pectinate muscles), 


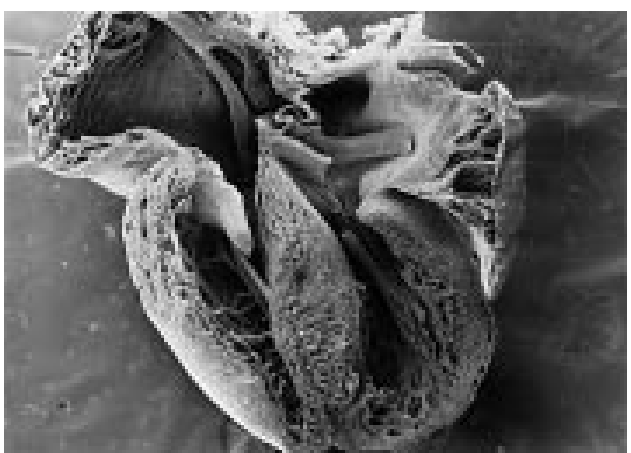

Figure 1 A coronal section of the heart showing trabeculated appendages in contrast to the smooth walled atrial bodies.

but these are less pronounced on the left. ${ }^{7}$ The extent of the pectinate muscles enables appendages to be designated as morphologically right or left (fig 1), a useful distinction in some cases of complex congenital heart disease.

\section{Comparative anatomy}

Until recently, there have been virtually no publications dealing with the comparative anatomy of the LAA. The first such detailed description of the varied morphology of the LAA was provided by Ernst et al, ${ }^{8}$ who studied the morphology of the LAA in 220 cases using synthetic resin casts made at necropsy. In 198 cases an antemortem ECG was available (143 in sinus rhythm and 55 in atrial fibrillation). LAA casts varied markedly in volume, from 0.7 $\mathrm{ml}$ to $19.2 \mathrm{ml}$. The size of the orifice of the LAA also varied considerably, with a minimum diameter ranging from 5 to $27 \mathrm{~mm}$, and a maximum diameter from 10 to $40 \mathrm{~mm}$. Likewise, there was great variation in the length of the LAA, which ranged from 16 to $51 \mathrm{~mm}$. In $70 \%$ of the cases, the principal axis was markedly bent or spiral, which accounts for some of the differences in shape and size of the LAA in vivo when viewed with transoesophageal echocardiography in different imaging planes. $^{9}$

Casts from patients who had been in atrial fibrillation were more voluminous, had larger orifices and fewer branches, and were broader than casts from patients who had been in sinus rhythm. Similar observations regarding LAA size in patients with atrial fibrillation have been made using transoesophageal echocardiography. ${ }^{10}$ It is not clear from these studies whether these differences are the result of the haemodynamic changes associated with atrial fibrillation or of the atrial fibrillation itself.

Ultrastructure and anatomical relations

The endocardium - It is currently not known whether the endocardium of the LAA is similar in structure and function to the rest of the heart.

The myocardium-The cardiac muscle cell structure of the appendages is similar to that of myocardium elsewhere. ${ }^{11}$

The epicardium and pericardium-The epicardium on the surface of the atria is thicker than over the ventricles. The LAA lies within the

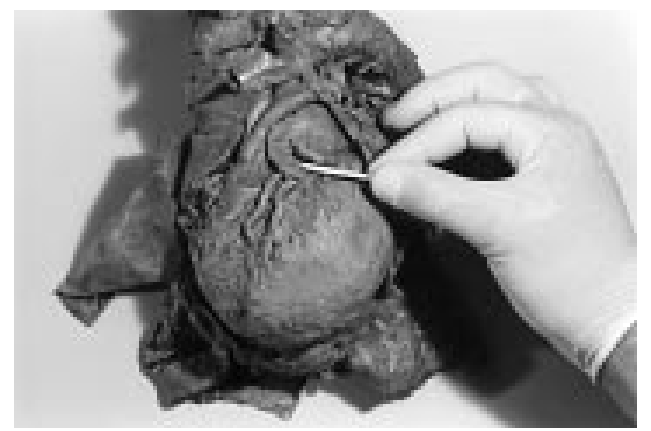

Figure 2 Picture of a gross specimen of the heart with the pericardium. The position of the appendage between the left ventricle and pulmonary artery and in close relation to the pericardium can be appreciated. The operator's hand is emphasising a prominent branch, which is narrow and has an irregular edge.

confines of the pericardium and is closely related in its superior aspect to the pulmonary artery and inferiorly to the free wall of the left ventricle (fig 2).

Blood supply - For practical purposes one may think of the entire atrial coronary circulation as being provided by the left circumflex and right coronary arteries from their course in the left and right atrioventricular sulci. ${ }^{12}$

Innervation - The atria are innervated by both sympathetic and parasympathetic fibres. Myelinated or unmyelinated afferent nerve fibres pass either in the vagus nerves to the brainstem or through sympathetic afferents to the spinal cord. ${ }^{13}$ Details on the neurophysiology of the LAA are discussed later.

\section{Physiology}

FLOW

The LAA shortens to a greater extent than the rest of the left atrium and has a distinct pattern of contraction. Blood flow within the LAA has been studied with transoesophageal echocardiography, which affords good views of the appendage and its orifice. Doppler measured LAA flow in patients with sinus rhythm was initially described as biphasic, ${ }^{10}{ }^{14}$ but additional emptying and filling waves resulting in quadriphasic appendage flow have been described in $40-70 \%$ of patients. ${ }^{15-17}$ This pattern is seen less often in patients undergoing transoesophageal echocardiography for clinical indications than in normal volunteers. The cycle begins with a phase of forward flow (out of the appendage) occurring soon after the start of transmitral flow in early diastole, followed by a short phase of backward flow (into the appendage). The first forward phase is fixed to the start of early diastole, suggesting a causal relation between left ventricular relaxation and early appendageal emptying. Coincident with atrial systole is a further phase of forward flow owing to contraction of the appendage, followed by another phase of backward flow possibly caused by elasticity of the appendage (fig 3 ). This sequence is constant and largely independent of heart rate.

The LAA lies within the fixed, relatively immobile confines of the pericardium. It is closely related in its superior aspect to the pul- 


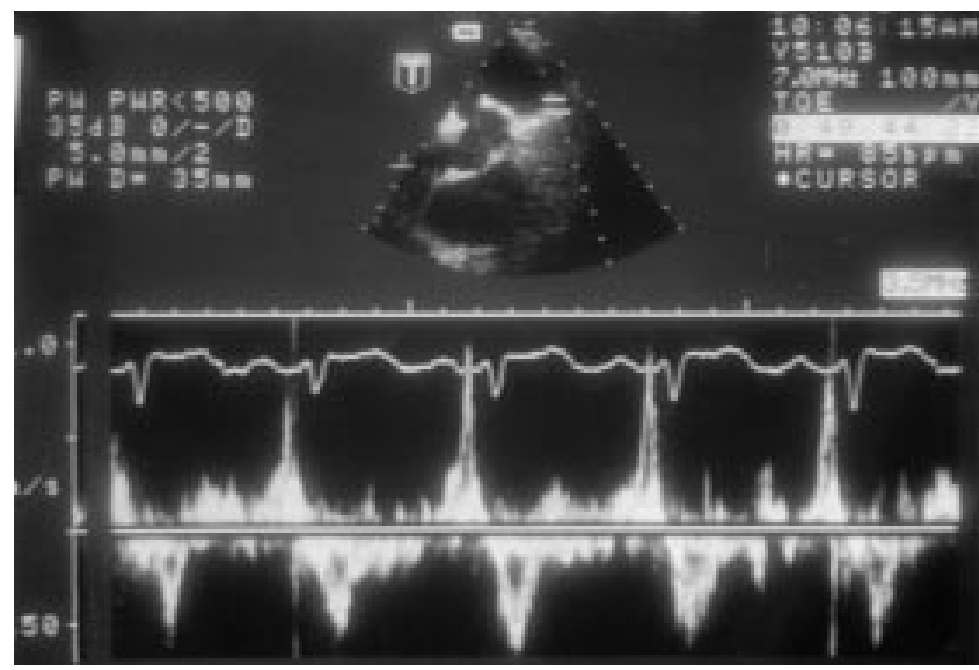

Figure 3 Flow in and out of the appendage during sinus rhythm. A complex quadriphasic pattern can be appreciated. Two inflow (below line) and two outflow (above line) phases are present. During atrial systole (after the P wave) a clear period of flow out of the appendage is seen. This is followed by flow into the appendage during ventricular systole (QRS complex). Two further waves are seen: an outflow wave (ventricular relaxation), and an inflow wave (probably related to appendageal elasticity).

monary artery and inferomedially to the free wall of the left ventricle. As the left ventricle dilates during diastole, it fills the intrapericardial space, and it has been suggested ${ }^{18}$ that it may contribute to appendageal emptying by compressing the inferomedial wall of the LAA between its free wall and the fixed pericardium. In addition, ventricular filling creates an intracavitary suction effect, influencing emptying and filling of the atrium and consequently the appendage. The extent of emptying and filling of the LAA may be influenced more by changes in left ventricular than LAA function, a finding which could in part help to explain the higher incidence of atrial fibrillation related stroke in patients with left ventricular dysfunction. ${ }^{19}$ The magnitude of LAA filling and emptying in atrial fibrillation may be inversely related to ventricular rate, a finding that could have implications for optimal rate control in preventing thromboembolism in atrial fibrillation. ${ }^{18} 20$

The relation of LAA flow to left ventricular filling also raises the question as to whether the appendage actively contracts or whether it functions passively, being compressed by the left ventricle during ventricular diastole and emptied by the negative pressure of ventricular filling. Evidence supports active contraction of the appendage, as it has prominent muscle ridges which, unless they contract, would atrophy. Furthermore, passive emptying and filling of the appendage would not easily explain the quadriphasic appendage flow seen at slower heart rates. In addition a case report ${ }^{21}$ of a herniated giant LAA records vigorous contraction during which the "distal tip would flip in an arch of approximately 3 inches with each systole." Finally, surgeons viewing the LAA at the time of surgery observe active contraction.

Several animal studies suggest that the LAA is more distensible than the left atrium proper. ${ }^{22-25}$ In humans, clamping of the LAA during cardiac surgery results in an increase in left atrial pressure and dimension as well as in transmitral and pulmonary diastolic flow velocities. ${ }^{26}$ Because of its increased distensibility, the LAA may augment haemodynamic function by modulating left atrial pressurevolume relations in states of increased left atrial pressure and volume overload. ${ }^{25}$

ATRIAL NATRIURETIC FACTOR

In humans, analysis of ANF content in excised atrial appendages has shown that they contain about $30 \%$ of all the cardiac ANF. The cardiocytes of the LAA contain the greatest density of ANF granules found in the left atrium. ${ }^{27} \mathrm{ANF}$ forms the major protein in isolated atrial amyloidosis and it is therefore of interest that this disease has a predilection for the LAA. ${ }^{28} 29$

\section{NEUROPHYSIOLOGY}

The atria and appendages are supplied by a variety of nerves and receptors. Both appendages can be selectively distended by infusing fluid into small balloons located within them without altering the pressures in the right or left atrium or aorta, ${ }^{30}$ resulting in an increase in urine flow and sodium excretion. However, crushing the bases of both appendages abolishes or attenuates this effect, suggesting that the response is initiated primarily by stretch sensitive receptors in the atrial appendages. In dogs, this also causes an increase in heart rate (a reflex response with a vagal afferent path and a sympathetic efferent path). ${ }^{30}$ Therefore it is possible that the LAA maintains left atrial pressure not only through its increased distensibility and its capacity for ANF secretion, but also through stretch sensitive receptors which, when activated, increase heart rate, diuresis, and natriuresis.

\section{Imaging left atrial appendage thrombus}

From a radiological perspective the LAA forms part of the normal cardiac silhouette on the left cardiac border between the left ventricle and pulmonary outflow tract. Although angiography has been used fairly extensively in the past for the investigation of thrombi in the LAA, ${ }^{31}$ this is invasive and is now rarely done. Transthoracic echocardiography does not usually afford views of the LAA; however, transoesophageal echocardiography allows semi-invasive, highly accurate imaging of the LAA and in recent years this has become a valuable tool for the diagnosis of thrombus within the appendage (figs 4 and 5). In a comparison with intraoperative observations, the sensitivity and specificity of transoesophageal echocardiography were $100 \%$ and $99 \%$, respectively, with a positive predictive value of $86 \%$ and a negative predictive value of $100 \%$ for the diagnoses of thrombi within the LAA. ${ }^{32}$

Current anticoagulation guidelines for cardioversion in patients with atrial fibrillation lasting for more than 48 hours recommend at least three weeks of warfarin treatment before cardioversion and a minimum of four weeks afterwards. ${ }^{33}$ An alternative approach would be to perform transoesophageal echocardiography and progress to immediate cardioversion without anticoagulation if no thrombi are present. ${ }^{34}$ This would minimise the duration of atrial 


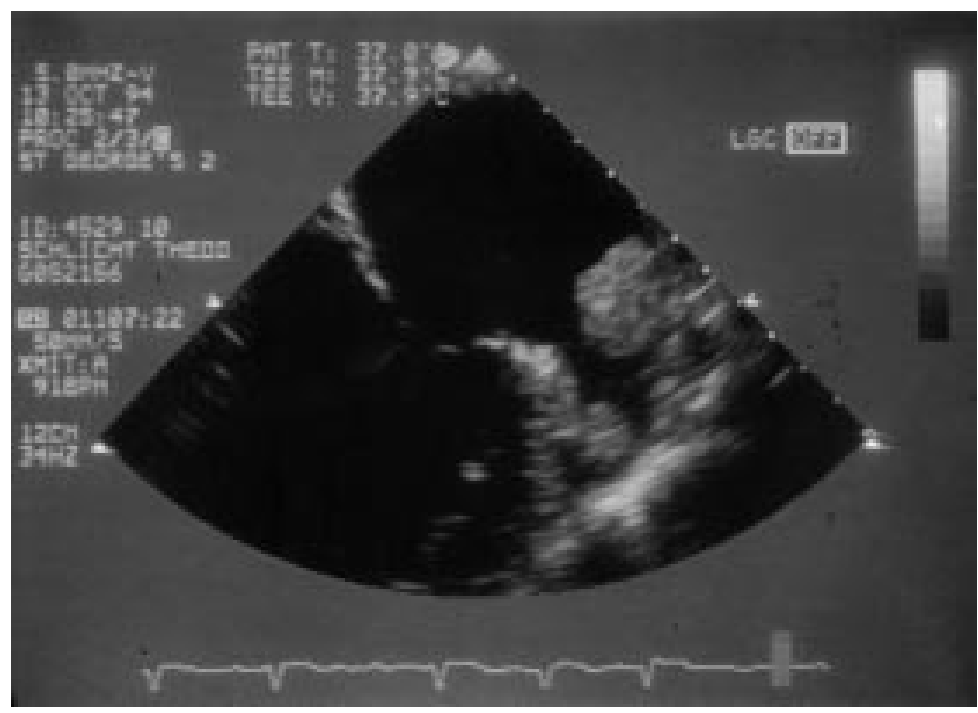

Figure 4 Left atrial appendage thrombi in a patient in atrial fibrillation. A small thrombus is present within the lumen of the appendage, and a larger one appears to be more fixed, and is present at the orifice.

fibrillation before cardioversion and increase the likelihood of success. ${ }^{35}$ It has been shown, however, that this strategy is suboptimal as $2-3 \%$ of patients without LAA thrombus on transoesophageal echocardiography before cardioversion still have an embolic event shortly after restoration of sinus rhythm. ${ }^{36}$ This problem may be overcome by a period of anticoagulation after cardioversion, and a large multicentre study (the ACUTE study (assessment of cardioversion using transoesophageal echocardiography)) is under way to evaluate the feasibility, safety, and cost-effectiveness of this approach.

\section{Correlates of left atrial appendage thrombus}

The LAA is the site most commonly associated with thrombus formation, particularly in patients with non-valvar atrial fibrillation. The pathogenesis of LAA thrombus has not been fully elucidated, but the predilection for its formation in the LAA is likely to result from

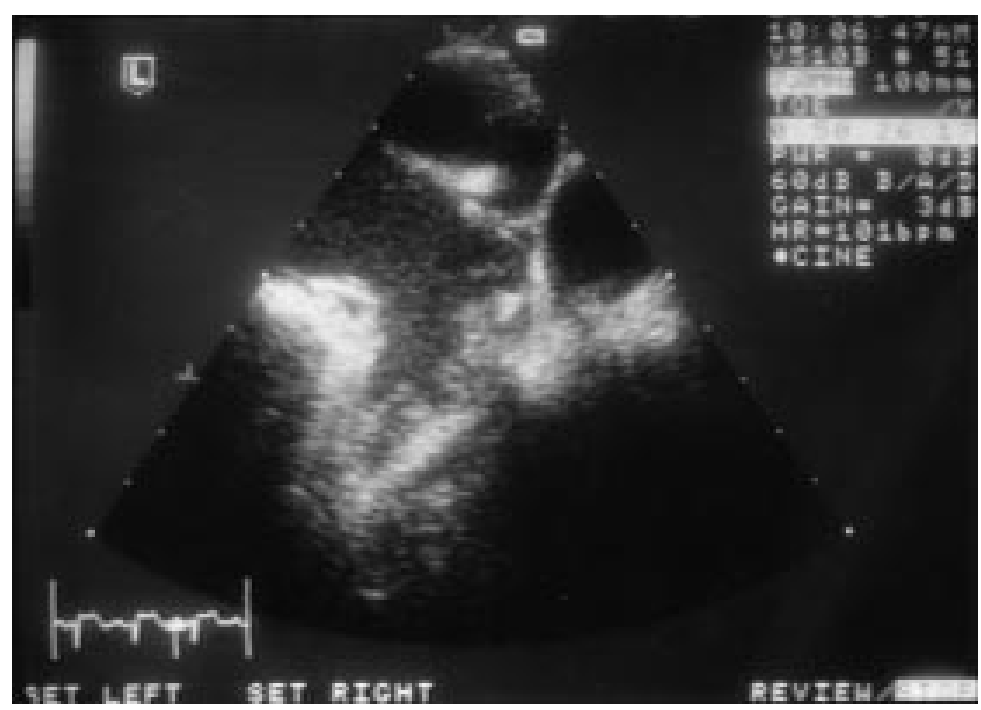

Figure 5 Prominent trabeculations within the left atrial appendage can easily be misinterpreted as thrombi. stagnation within the long, blind ended trabeculated pouch. Transoesophageal echocardiography on 233 patients with atrial fibrillation of more than 48 hours' duration who were not on chronic anticoagulation revealed that $15 \%$ had evidence of left atrial thrombus, and in all but one case this was located in the appendage. ${ }^{37}$ In another series of 272 patients with non-rheumatic atrial fibrillation, the prevalence of thrombi detected at transoesophageal echocardiography was $8 \%$ and all were in the appendage. ${ }^{38}$ Less commonly, intra-atrial thrombi also form in patients with atrial flutter and were detected at transoesophageal echocardiography in five of 24 patients with atrial flutter; again, all thrombi were located in the LAA. ${ }^{39}$ Three specific variables of LAA structure and function have been studied with respect to thrombus formation and stroke: size, flow pattern, and flow velocity.

\section{LAA SIZE}

There has been controversy over whether the size of the LAA determines thromboembolic risk. ${ }^{40-42}$ However, LAA size determined at surgery has been related to the occurrence of embolic events ${ }^{41}$ and studies with transoesophageal echocardiography have shown that thrombus and subsequent thromboembolic events are clearly related to LAA size. An enlarged left atrium on transoesophageal echocardiography is associated with an enlarged LAA in $59 \%$ of patients; conversely, a normal sized left atrium is associated with an enlarged LAA in $15 \%$ of patients. Spontaneous echo contrast, or smoke-like echo, has been defined as an amorphous, swirling, light grey haze inside the cardiac chambers, great vessels, and veins. It arises in conditions of stasis, has been attributed to rouleaux formation, and is strongly associated with LAA thrombus formation. Left atrial size is larger in patients with spontaneous echo contrast and thrombosis in the LAA than in those without these features (52.1 (9.0) $\mathrm{mm} v 39.6$ (11.0) $\mathrm{mm}$, mean (SD)). LAA area has been shown to be larger in patients who have evidence of LAA thrombus, whether they are in atrial fibrillation or in sinus rhythm. ${ }^{10}$ In patients with recent onset atrial fibrillation, LAA thrombus is associated with a large LAA area, ${ }^{43}$ and in chronic atrial fibrillation it has also been shown, using intravenous albumin as a contrast agent, that LAA area is significantly larger in patients with thrombus than in those without. ${ }^{44}$

LAA FUNCTION AND FLOW PATTERNS

Doppler transoesophageal echocardiography has been used extensively in recent years to determine the velocity and pattern of blood flow at the orifice of the appendage (fig 6).

Reduced or absent LAA inflow and outflow velocities and low LAA ejection fractions are associated with LAA spontaneous echo contrast and thrombus formation in patients in sinus rhythm and in those in atrial fibrillation, ${ }^{9} 10144546$ and low peak LAA filling and emptying velocities have been found to be related to a history of systemic embolism. ${ }^{47}$ 


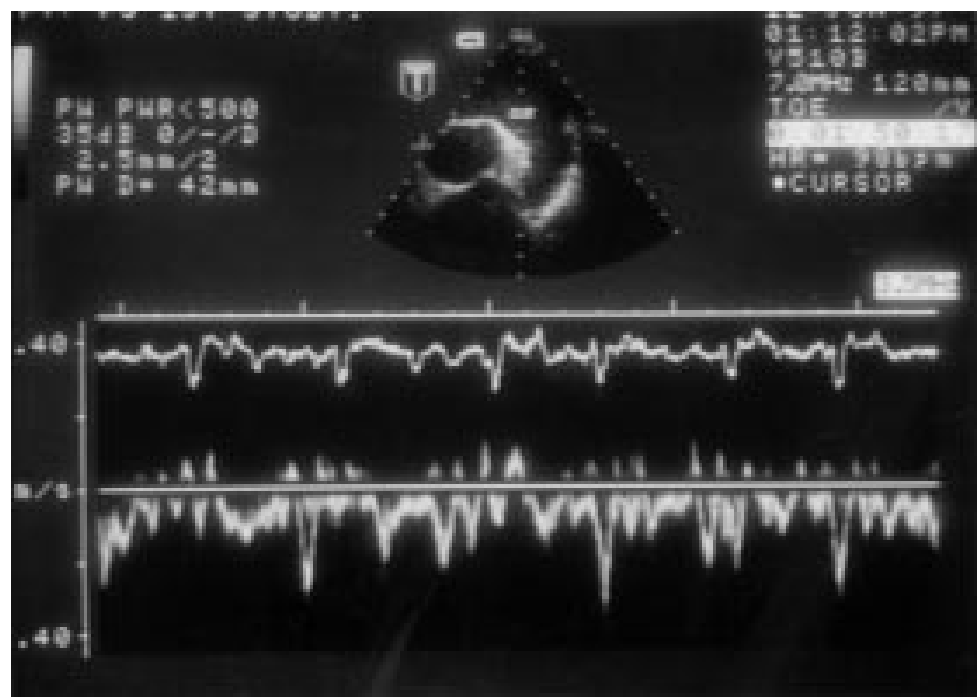

Figure 6 Left atrial appendage flow during atrial fibrillation, as determined by pulsed Doppler during transoesophageal echocardiography. Both the velocity and the pattern of flow have been related to the formation of thrombi and to the development of subsequent thromboembolic events. In this example, a well defined flow pattern is present and the maximum inflow velocity is approaching $0.4 \mathrm{~m} / \mathrm{s}$, a relatively high velocity. Thus the risk of thrombus formation in this patient would be relatively low.

LAA flow patterns have been classified into three types according to the appearance on Doppler transoesophageal echocardiography ${ }^{14}$ : Type I-patients in sinus rhythm with a regular pattern of filling and emptying;

Type II-patients in atrial fibrillation with an active "sawtooth" pattern;

Type III-patients in atrial fibrillation with no identifiable flow waves.

Patients with the type III pattern have a significantly higher incidence of LAA spontaneous echo contrast and thrombus than those with the type II or I pattern. LAA flow patterns have been related to the appearance of fibrillatory waves on the ECG. ${ }^{48}$ Patients with coarse fibrillatory waves had lower peak LAA emptying velocity and ejection fraction and a higher incidence of LAA spontaneous echo contrast and thrombus than those with fine fibrillatory waves. The cause of LAA dysfunction in patients with atrial fibrillation has not been fully elucidated. It is likely to be related in part to a myopathic process which results in atrial fibrillation or which occurs as a result of the atrial fibrillation itself (an atrial fibrillation induced myopathy). LAA function in patients with atrial flutter is reported to be associated with a regular pattern of LAA emptying and significantly higher peak emptying velocities than in patients with atrial fibrillation, ${ }^{49}$ in keeping with the lower incidence of thromboembolism in atrial flutter. ${ }^{50}$

The size and mobility of the thrombus are also determinants of thromboembolic risk. Thrombus dimensions of more than $1.5 \mathrm{~cm}$ and thrombi that are pedunculated and mobile are associated with increased risk of thromboembolism. ${ }^{51}$

Patients with atrial fibrillation who are successfully cardioverted with drugs or dc shock show depressed left atrial mechanical function for a variable period following restoration of sinus rhythm. Similar findings have been reported in studies on LAA function.
LAA spontaneous echo contrast develops de novo, and inflow and outflow velocities in the appendage decrease after successful cardioversion of atrial fibrillation. ${ }^{152}$ These findings are important, as depressed LAA function may result in thrombus formation. The finding of decreased atrial mechanical function after cardioversion is sometimes referred to as "stunning"; however, this term is a misnomer as it implies that the dc shock itself results in damage to the atrium and appendage with consequent depressed function and stasis. This is unlikely to be the case as studies on patients have shown that the phenomenon occurs after both pharmacological ${ }^{53}$ and spontaneous cardioversion of atrial fibrillation, ${ }^{54}$ and shocks have not been shown to cause atrial mechanical dysfunction in patients cardioverted for ventricular tachycardia. ${ }^{55}$ Decreased atrial mechanical function after cardioversion may occur as a result of an atrial fibrillation induced atrial and appendageal myopathy as the depression of function is related to the duration of the previous atrial fibrillation. ${ }^{37}$

\section{LAA THROMBUS IN PATIENTS IN SINUS RHYTHM}

Five per cent of patients in sinus rhythm with a history of stroke, transient ischaemic attack, or systemic embolisation and no significant carotid stenosis have LAA thrombi. ${ }^{56}$ Significant left ventricular dysfunction in sinus rhythm is associated with left atrial thrombus formation, and about $15 \%$ of patients with severe dilated cardiomyopathy in sinus rhythm have identifiable atrial thrombi, ${ }^{57}$ the majority of which are located within the LAA. ${ }^{58}$ These and other data suggest that the atrial appendage may be a source of embolic material even in the absence of atrial fibrillation.

HAEMATOLOGICAL AND ENDOCARDIAL CORRELATES OF LAA THROMBUS

Thrombi are not fixed structures but are continuously forming and resolving. Hence left atrial thrombus is associated with raised peripheral blood concentrations of fibrinopeptide A, reflecting thrombin mediated fibrin formation, and $\mathrm{D}$ dimer, a degradation product of fibrinogen. ${ }^{59}$ The observation that warfarin treatment results in disappearance of LAA thrombi when patients are re-examined with transoesophageal echocardiography after four weeks of therapy further suggests that the presence of thrombus is the result of a dynamic process of clot formation and lysis. The relative role of platelets and endothelium in the pathophysiology of LAA thrombus formation is not known.

\section{Obliteration of the left atrial appendage} for the prophylaxis of thromboembolism Randomised trials have shown that anticoagulation with warfarin results in a significant reduction in stroke rate and mortality in patients with atrial fibrillation. The effects of aspirin are far more modest. It should be noted that in these trials anticoagulated patients were highly selected (in three of the North American warfarin/placebo trials, only about $10 \%$ were 
ultimately randomised). In those assigned to warfarin, withdrawals occur at rates of up to $38 \%$ a year. $^{60}$ In warfarin treated patients, approximately $50 \%$ of the strokes occurred in individuals who had inadvertent therapeutic lapses, or who required temporary or permanent cessation of therapy, ${ }^{60-62}$ a finding that parallels experience in patients with valvar prostheses. ${ }^{63} 64$

More than $50 \%$ of the atrial fibrillation population are aged 75 or older ${ }^{62}$ and it has been estimated that $20 \%$ or more have a contraindication to warfarin treatment. ${ }^{65}$ There is not only underprescribing of warfarin to patients who are eligible, but also a high incidence of contraindications in patients who are at risk of thromboembolism. Thus, although warfarin is effective in reducing the incidence of stroke, in practice it is often difficult to treat patients continuously and effectively in the recommended manner.

LAA obliteration as an alternative means of stroke prophylaxis is attractive, as over $90 \%$ of thrombi associated with non-rheumatic atrial fibrillation occur predominantly within the LAA. Data from the placebo arm of the European atrial fibrillation trial suggest that in patients who have had a previous stroke and have contraindications to warfarin treatment, the annual stroke rate is $12 \%$ a year. It is estimated that if obliteration reduces the incidence of stroke by $50 \%$ to $75 \%$, this high stroke rate could be reduced to $3-6 \%$ a year. ${ }^{5}$ This is similar to findings reported in the North American symptomatic carotid endarterectomy trial. ${ }^{66}$ There are thus two possible situations in which amputation or obliteration of the LAA may be considered: first, as an additional procedure during cardiac surgery in patients in whom warfarin is required but is contraindicated; and second, as an isolated closed chest (for example thoracoscopic) procedure.

In 1952 Bailey et al suggested that the LAA could be amputated at mitral valvotomy as a means of prophylaxis of thromboembolism. ${ }^{67} \mathrm{~A}$ few years later Belcher and Somerville added support for this strategy and advocated surgical obliteration of LAA when it was large.$^{68}$ It was suggested that this procedure could offer approximately $50 \%$ protection against the risk of stroke even in patients with mitral valve disease.

Amputation of the LAA remains controversial, although excision or ligation of the right atrial appendage is frequently performed during insertion or withdrawal of cannulae during coronary artery bypass grafting. A recent review suggested that surgeons use the technique of LAA obliteration sporadically. ${ }^{1}$ Some obliterate the appendage during mitral valve replacement and repair, while others use it only as part of the maze or corridor procedures for the surgical treatment of atrial fibrillation. Various techniques for the intraoperative amputation of the LAA have been described, including the use of an automatic surgical stapler. ${ }^{69}$

LAA obliteration may result in adverse haemodynamic and physiological effects. Various animal models have been used to examine the effects of amputation of the atrial appendages on natriuresis and diuresis in response to volume loading. In the monkey, bilateral appendectomy greatly attenuates the rise in plasma $\mathrm{ANF}$ and attenuates natriuresis after acute volume expansion. ${ }^{70}$ Atrial appendectomy also results in a reduction in stroke volume, which may be explained by a reduction in atrial "kick." Bilateral atrial appendectomy in conscious dogs eliminates ANF release and blunts renal excretion of sodium and water after a large acute volume load. ${ }^{71}$ Similar findings have been reported in human subjects. ${ }^{72}$ It may be possible to diminish the hormonal effects of LAA obliteration by preserving the right atrial appendage.

In addition to the effects on ANF secretion, amputation of the LAA may also have undesirable effects on atrial compliance. Removal of the appendage in open chested dogs causes a decrease in atrial compliance associated with altered left ventricular filling and atrial systolic function, ${ }^{22}$ and in patients undergoing cardiac surgery, the mean left atrial pressure and dimension and transmitral and pulmonary diastolic flow velocities rose after LAA clamping. ${ }^{22}$

Thus before the routine use of LAA obliteration during cardiac surgery can be advocated or novel treatments such thoracoscopic LAA obliteration proposed, the haemodynamic and hormonal effects of such a manoeuvre must be carefully evaluated.

\section{Conclusions}

The LAA has unique developmental, anatomical, and physiological properties. These properties render it ideal to act as a reservoir in conditions of volume overload, and to effect the adaptive responses necessary for the reduction of circulating blood volume. The LAA is the major site of thrombus formation in non-valvar atrial fibrillation and to a lesser extent in mitral valve disease. Although the exact pathogenesis of this complication has not been fully elucidated, it is likely that stasis of blood flow within the LAA plays a major role, but much is unknown. In particular, the role of the endothelium of the LAA and the haematological mechanisms of LAA thrombus formation have not been adequately studied.

The most effective current prophylaxis of stroke in atrial fibrillation is warfarin. Warfarin is contraindicated in many patients, particularly in the elderly in whom the risk of stroke is highest. Alternative treatments are needed, and obliteration of the LAA is one such option. However, this is technically challenging and may result in unfavourable haemodynamic and hormonal effects, which could be particularly important in patients with left ventricular failure and valvar heart disease. Direct or thoracoscopic obliteration are possibilities, but technical aspects of the thoracoscopic procedure must be developed before it can be offered as an alternative. The technique will not prevent all episodes of thromboembolism, particularly in patients with mitral valve disease, in whom 
the appendage is the sole location of thrombus in only $60 \%$ of patients.

Future research will explore alternative treatments for thromboprophylaxis in atrial fibrillation and will determine whether LAA obliteration results in effective prophylaxis against thromboembolism in humans, and whether clinically significant adverse effects result from such a procedure.

We thank Dr Sandra Webb, Anatomy Department, and $\mathrm{Mr}$ Raymond Moss, Electron Microscopy Unit, St George's HospiRaymond Moss, Electron Microsc
tal Medical School, for figure 1 .

1 Blackshear JL, Odell JA. Appendage obliteration to reduce stroke in cardiac surgical patients with atrial fibrillation. Ann Thorac Surg 1996;61:755-9.

2 Wolf PA, Abbott RD, Kannel WB. Atrial fibrillation as an independent risk factor for stroke: the Framingham study. Stroke 1991;22:983-8.

3 Wolf PA, Dawber TR, Thomas HE, et al. Epidemiological assessment of chronic atrial fibrillation and risk of stroke: The Framingham study. Neurology 1978;28:973-7.

4 Kannel W, Wolf PA. Epidemiology of atrial fibrillation. In Falk RH, Podrid PJ, eds. Atrial fibrillation: mechanisms and management. New York: Raven Press, 1992:81-92.

5 Odell JA, Blackshear JL, Davies E, et al. Thoracoscopic obliteration of the left atrial appendage: potential for stroke reduction. Ann Thorac Surg 1996;61:565-9.

6 Brass LM, Krumholz HM, Scinto JM, et al. Warfarin for use among patients with atrial fibrillation. Stroke 1997;28 2382-9.

7 Sadler TW. Cardiovascular system. In: Langman J, ed. Langman's medical embryology, 6th ed. Baltimore: Williams and Wilkins, 1990:179-227.

8 Ernst G, Stollberger C, Abzieher F, et al. Morphology of the left atrial appendage. Anat Rec 1995;242:553-61.

9 Mugge A, Kunn H, Nikutta P, et al. Assessment of left atrial appendage function by biplane transesophageal echocardiography in patients with non rheumatic atrial cardiography in patients with non rheumatic atrial fibrillation: identification of a subgroup of patients at
increased embolic risk. F $\mathrm{Am}$ Coll Cardiol 1994;23:599incre 607 .

10 Pollick C, Taylor D. Assessment of left atrial appendage function by transesophageal echocardiography. Implications for the development of thrombus. Circulation $1991 ; 84: 223-31$

11 Lannigan RA, Zaki SA. Ultrastructure of myocardium of the atrial appendage. Br Heart $\mathcal{F}$ 1966;28:796-807.

12 James TN. Small arteries of the heart. Circulation 1977;56: $2-14$.

13 Bishop VS, Malliani A, Thoren P. Cardiac mechanoreceptors. Peripheral circulation and organ blood flow. In: Shepherd JT, Abboud FM, eds. Handbook of physiology. Washington DC: American Physiological Society; 1983:497-555.

14 Garcia-Fernandez MA, Torrecilla EG, Roma DS, et al. Left atrial appendage Doppler flow patterns: implications on atrial appendage Doppler flow patterns: implications
thrombus formation. Am Heart f 1992;124:955-61.

15 Grimm RA, Stewart WJ, Maloney JD, et al. Impact of electrical cardioversion for atrial fibrillation on left atrial appendage function and spontaneous echo contrast: appendage function and spontaneous echo contrast: characterisation by simultaneous transesophageal

16 Jue J, Winslow T, Fazio G, et al. Pulsed Doppler characterization of left atrial appendage flow. $\mathcal{F} \mathrm{Am}$ Soc Echocardiogr 1993;6:237-44.

17 Mikael Kortz RA, Delemarre BJ, van Dantzig JM, et al. Left atrial appendage blood flow determined by transesophageal echocardiography in healthy subjects. $\mathrm{Am} \mathcal{F}$ Cardio 1993;71:976-81.

18 Akosah KO, Funai JT, Porter TR, et al. Left atrial appendage contractile function in atrial fibrillation. Influence of heart rate and cardioversion to sinus rhythm. Chest 1995;107:690-6.

19 The Stroke Prevention in Atrial Fibrillation Investigators. Predictors of thromboembolism in atrial fibrillation: II. Echocardiographic features of patients at risk. Ann Intern Echocardiographic

20 Hoit BD, Shao Y, Gabel M. Influence of acutely altered loading conditions on left atrial appendage flow velocities. $\mathcal{f}$ Am Coll Cardiol 1994:24:1117-23.

21 Dimond EG, Kittle CF, Voth DW. Extreme hypertrophy of the left atrial appendage. The case of the giant dog ear. $\mathrm{Am}$ f Cardiol 1960;5:122-5.

22 Hoit BD, Shao Y, Tsai LM, et al. Altered left atrial compliance after atrial appendectomy. Influence on left atrial and ventricular filling. Circ Res 1993;72:167-75.

23 Hoit BD, Walsh RA. Regional atrial distensibility. Am f Physiol 1992;262:H1356-60.

24 Bauman RP, Rembert JC, Greenfield JC. Regional atrial blood flow in dogs. Effect of hypertrophy on coronary flow reserve. F Clin Invest 1989;83:1563-9.

25 Davis CA, Rembert JC, Greenfield JC. Compliance of left atrium with and without left atrium appendage. $A m \mathcal{F}$ Physiol 1990;259:H1006-8.

26 Tabata $\mathrm{T}$, Oki $\mathrm{T}$, Yamada $\mathrm{H}$, et al. Roles of left atrial appendage in left atrial reservoir function as evaluated by left atrial appendage clamping during cardiac surgery. $A m \mathcal{F}$ Cardiol. 1998;81:327-32.
27 Chapeau C, Gutkowska J, Schiller PW, et al. Localization of immunoreactive synthetic atrial natriuretic factor (ANF) in the heart of various animal species. 7 Histochem Cytochem 1985;33:541-50.

28 Kaye GC, Butler MG, D'Ardenne AJ, et al. Identification of immunoreactive atrial natriuretic peptide in atrial amyloid. f Clin Pathol 1986;39:581-2

29 Linke RP, Voigt C, Strorkel FS, et al. N-terminal amino acid sequence analysis indicates that isolated atrial amyloid is derived from atrial natriuretic peptide. Virchows Arch B Cell Pathol Mol Pathol 1988;:125-7.

30 Kappagoda CT, Linden RJ, Saunders DA. The effect on heart rate of distending the atrial appendages in dogs. $f$ Physiol (Lond) 1972;225:705-19.

31 Sakomoto I, Hayashi K, Matsunaga N, et al. Coronary angiographic finding of thrombus in the left atrial appendage. Acta Radiol 1996;37:749-53.

32 Manning WJ, Weintraub RM, Waksmonski CA, et al. Accuracy of transesophageal echocardiography for identifying left atrial thrombi. A prospective, intraoperative study. Ann Intern Med 1995;123:817-22.

33 Anderson JL. Acute treatment of atrial fibrillation and flutter. Am f Cardiol 1996;78:17-21.

34 Salka S, Saeian K, Sagar KB. Cerebral thromboembolization after cardioversion of atrial fibrillation in patients without transesophageal echocardiographic findings of left atrial thrombus. Am Heart f 1993;126:722-4

35 Manning WJ, Silverman DI, Gordon SP, et al. Cardioversion from atrial fibrillation without prolonged anticoagulation with use of transesophageal echocardiography to exclude the presence of atrial thrombi. N Engl F Med 1993;328: $750-5$.

36 Black IW, Fatkin D, Sagar KB, et al. Exclusion of atrial thrombus by transesophageal echocardiogrphy does not preclude embolism after cardioversion of atrial fibrillation. A multicenter study. Circulation 1994:89:2509-13.

37 Manning WJ, Siverman DI, Katz SE, et al. Impaired left atrial mechanical function after cardioversion: relation to the duration of atrial fibrillation. I Am Coll Cardiol 1994;23:1535-40

38 Leung DY, Black IW, Cranney GB, et al. Prognostic implications of left atrial spontaneous echo contrast in nonvalvular atrial fibrillation. F Am Coll Cardiol 1994;24: $755-62$

39 Bikkina M, Alpert MA, Mulekar M, et al. Prevalence of intraatrial thrombus in patients with atrial flutter. $A m \mathcal{F}$ Cardiol 1995;76:186-9.

40 Sommerville W, Chambers J. Systemic embolism in mitral stenosis: relation to the size of the left atrial appendix. $B M F$ 1964;ii: 1167-9.

41 Neilson GH, Galea EG, Hossack KF. Thromboembolic complications of mitral valve disease. Aust $N Z \mathcal{F} \mathrm{Med}$ 1978;8:372-6.

42 Fleming H, Bailey SM. Mitral valve disease, systemic embolism and anticoagulants. Postgrad Med F 1971;47:599-604

43 Rubin DN, Katz SE, Riley MF, et al. Evaluation of left atria appendage anatomy and function in recent-onset atrial fibrillation by transesop
Cardiol $1996 ; 78: 744-78$

44 Kato H, Nakanishi M, Maekawa N, et al. Evaluation of left atrial appendage stasis in patients with atrial fibrillation using transesophageal echocardiography with an intravenous albumin-contrast agent. Am f Cardiol 1996;78:365-9.

45 Pozzoli M, Febo O, Torbicki A, et al. Left atrial appendage dysfunction: a cause of thrombosis? Evidence by transesophageal echocardiography-Doppler studies. $\mathcal{f} \mathrm{Am}$ Soc Echocardiogr 1991;4:435-41.

46 Suetsugu M, Matsuzaki M, Toma Y, et al. Detection of mural thrombi and analysis of blood flow velocities in the left atrial appendage using transesophageal twodimensional echocardiography and pulsed Doppler flowmetry. $\mathcal{F}$ Cardiol 1988;18:385-94.

47 Verhorst PM, Kamp O, Visser CA, et al. Left atrial appendage flow velocity assessment using transesophageal echocardiology in nonrheumatic atrial fibrillation and systemic embolism. Am f Cardiol 1993;71:192-6.

$48 \mathrm{Li} \mathrm{YH}$, Hwang JJ, Tseng YZ, et al. Clinical significance of fibrillatory wave amplitude. A clue to left atrial appendage function in non-rheumatic atrial fibrillation. Chest 1995; 108:359-63.

49 Santiago D, Warshofsky M, Li Mandri G, et al. Left atrial appendage function and thrombus formation in atrial fibrillation-flutter: a transesophageal echocardiographic study. 7 Am Coll Cardiol 1994;24:159-64.

50 Wood KA, Eisenberg SJ, Kalman JM, et al. Risk of thromboembolism in chronic atrial flutter. Am 7 Cardiol 1997;79:1043-7.

51 Leung DY, Davidson PM, Cranney GB, et al. Thromboembolic risks of left atrial thrombus detected by transesophageal echocardiogram. Am F Cardiol 1997;79:626-9.

52 Fatkin D, Kuchar DL, Thorburn CW, et al. Transesophageal echocardiography before and during direct current cardioversion of atrial fibrillation: evidence for "atrial stunning" as a mechanism of thromboembolic complications. f Am Coll Cardiol 1994;23:307-16.

53 Falcone RA, Morady F, Armstrong WF. Transesophageal echocardiographic evaluation of left atrial appendage function and spontaneous contrast formation after chemical or electrical cardioversion of atrial fibrillation. Am $\mathcal{F}$ Cardiol 1996;78:435-9.

54 Grimm RA, Leung DY, Black IW, et al. Left atrial appendage "stunning" after spontaneous cardioversion of atrial fibrillation demonstrated by transesophageal Doppler echocardiography. Am Heart $\mathcal{F}$ 1995;130:174-6. 
55 Dodds GA, Wilkinson WE, Greenfield RA, et al. Evaluation of the effect of transthoracic cardioversion from ventricular tachycardia to sinus rhythm on the left atrial mechanical function. Am 7 Cardiol 1996;78:1436-9.

56 Labovitz AJ, for the STEPS Investigators. Transesophageal echocardiography in patients with unexplained cerebral ischaemia: multicenter findings [abstract]. Circulation 1994;90(suppl 1):21.

57 Vigna C, Russo A, De Rito V, et al. Frequency of the lef atrial thrombi by transesophageal echocardiography in idiopathic and in ischemic dilated cardiomyopathy. $A m \mathcal{F}$ Cardiol 1992;70:1500-1.

58 Siostrzonek P, Koppensteiner R, Gossinger H, et al. Hemodynamic and hemorrheologic determinants of the left atrial spontaneous echo contrast and thrombus formation in patients with idiopathic dilated cardiomyopathy. Am Heart 7 1993;124:430-4.

59 Scardi S, Mazzone C, Pandullo C, et al. Non rheumatic atrial fibrillation and left atrial thrombus formationrelation between left atrial appendage function, clinical and haematological findings [abstract]. 7 Am Coll Cardiol 1997; 29(suppl):526A.

60 Petersen P, Boysen G, Godtfredsen J, et al. Placebocontrolled, randomised trial of warfarin and aspirin for prevention of thromboembolic complications of atria fibrillation. The Copenhagen AFASAK Study. Lance 1989;i:175-9.

61 Atrial Fibrillation Investigators. Risk factors for stroke and efficacy of anti-thrombotic therapy in atrial fibrillation: analysis of pooled data from five randomised controlled trials. Arch Intern Med 1994;154:1449-57.

62 Albers GW. Atrial fibrillation and stroke: three new studies, three remaining questions. Arch Intern Med 1994;154: 1443-8.
63 Saour JN, Sieck JO, Mamo LA, et al. Trial of different intensities of anticoagulation in patients with prosthetic heart .

64 Jegaden O, Eker A, Delavahe F, et al. Thromboembolic risk and late survival after mitral valve replacement with the St Jude Medical Valve. Ann Thorac Surg 1994;58:1721-8.

65 Gottlieb LK, Salem-Schatz S. Anticoagulation in atrial fibrillation: does efficacy in clinical trials translate into effectiveness in practice? Arch Intern Med 1994;154:194553.

66 North America Symptomatic Carotid Endarterectomy Trial Collaborators. Beneficial effect of carotid endarterectomy in symptomatic patients with high-grade carotid stenosis. $N$ Engl f Med 1991;325:445-53.

67 Bailey CP, Olsen AK, Keown KK, et al. Commissurotomy for mitral stenosis: technique for prevention of cerebral complications. $7 A M A$ 1952;149:1085-91.

68 Belcher JR, Somerville W. Systemic embolism and left auricular thrombosis in relation to mitral valvotomy. BMf 1955;ii: $1000-3$.

69 DiSesa VJ, Tam S, Cohn LH. Ligation of the left atrial appendage using an automatic surgical stapler. Ann Thorac appendage using an autc $1988 ; 46: 652-3$.

70 Benjamin BA, Metzler CH, Peterson TV. Chronic atrial appendectomy alters sodium excretion in conscious monkeys. Am F Physiol 1988;254:R699-705.

71 Stewart JM, Dean R, Brown M, et al. Bilateral atrial appendectomy abolishes increased plasma atrial natriuretic peptide release and blunts sodium and water excretion during volume loading in conscious dogs. Circ Res 1992;70: 724-32.

72 Omari BO, Nelson RJ Robertson JM. Effect of right atrial appendectomy on the release of atrial natriuretic hormone. f Thorac Cardiovasc Surg 1991;102:272-9. 